\title{
Physically-Based Validation of Deformable Medical Image Registration
}

\author{
Huai-Ping Lee ${ }^{1}$, Ming C. Lin ${ }^{1}$, and Mark Foskey ${ }^{1,2}$ \\ ${ }^{1}$ Dept. of Computer Science, \\ ${ }^{2}$ Dept. of Radiation Oncology, University of North Carolina at Chapel Hill, USA \\ \{lhp, lin,foskey\}@cs.unc.edu \\ http://gamma.cs.unc.edu/DeformReg/
}

\begin{abstract}
We propose a new approach for validating deformable image registration algorithms. Since difference images do not necessarily reflect the $3 \mathrm{D}$ correspondence of organs, we propose to use the deformation fields generated in our FEM-based simulations to assess the displacement resulted from other registration methods. Unlike traditional FEM-based registration methods, the boundary condition for the target organ is not given explicitly. Instead it is driven by inter-organ contact forces generated by boundary conditions on surrounding organs to reduce the uncertainty induced by geometry-based surface matching. To validate our system, real CT images of the male pelvis are analyzed, and the prostate can be reasonably registered without matching its surface to the image. Several registration methods are then evaluated using our system.
\end{abstract}

\section{Introduction}

In image-guided radiation cancer therapy, it is essential to establish correspondence between images taken on different days in order to assess the accumulated dosage. While rigid image registration is relatively easy, deformable registration is often the missing key for good correspondence. Image analysis techniques such as optical flow 12 or feature tracking [3 have been used to drive the deformations. Optical flow, however, may fall into local minima, and feature tracking requires local features that are not always easy to find. Some methods rely on minimizing an energy function based on image difference 445. If the optimization or matching process only makes use of a sparse set of points, the deformation field can be interpolated using simple radial basis functions [6] or physically-inspired basis functions [7. Model fitting on segmented images can also give a deformation [8], but they only fit surface vertices, so the accuracy depends on the interpolation method. Image- or model-based methods are not necessarily physically accurate [9].

Several physically-based models have been applied to registration, such as the viscous fluid model [105], which can handle large deformations, but fluidlike deformations are not always realistic for human organs. The finite element method (FEM) and the linear elasticity model [1] have been introduced into medical image analysis 12 and registration [1314 15 16]. Organs are modeled

D. Metaxas et al. (Eds.): MICCAI 2008, Part II, LNCS 5242, pp. 830-838, 2008.

(C) Springer-Verlag Berlin Heidelberg 2008 
as elastic bodies, and the boundary conditions in the domain can be the gradient field [13], or computed from fitted models [1415]. FEM-based methods can model the material properties better than the fluid flow methods and can provide a more global solution to the entire domain, but the accuracy is dependent upon the surface matching algorithm and the estimation of material properties.

The validation of registration algorithms has been limited to using image differences, but our goal is to assess the physical accuracy of 3D deformations of organs, not merely the intensity match of $2 \mathrm{D}$ images. Also, there is a lack of a common set of testing images. Arbitrarily deformed images are often used for testing, but they are not physically accurate. An FEM-based validation method has been proposed [9], in which a deformation field produced by another registration method is fed into the linear system generated during FEM simulations, and the external forces on non-boundary nodes can be an estimation of the quality of the deformation field.

In this paper, we propose using the deformation field generated by our multiorgan FEM system to generate realistic test images for registration methods. A volumetric mesh is built for the entire region of interest, including all organs and the tissue between them. We focus on the prostate and nearby organs because it is a site where organ motion is of particular concern. We do not apply boundary conditions on target organs to avoid the uncertainty induced by surface matching. The organs without explicit boundary conditions are deformed by inter-organ contact forces during simulation. This approach is similar to the one proposed by Hensel et al. [16, but they apply a translation to surrounding organs to align the prostate prior to deformable registration in order to cope with extreme deformations due to insertion of the endorectal coil, and the bladder and the rectum are modeled as solid objects, while we model them as hollow objects to reflect their actual structure. Our physical model is more accurate partly due to the fact that the deformation of the organs is not extreme and somewhat constrained by inter-organ interaction as observed in a human body. To verify our system, we perform an experimental registration. Although the model of the prostate itself is not fitted to the real image, the resulting deformation can register the model quite well. We then use our method to perform a basic evaluation of several registration algorithms. A set of physically accurate deformation fields and synthetic test images are generated with the simulator. The deformation field produced by the registration method is compared against the one that generated the test image to assess the accuracy of the registration.

\section{Method}

\subsection{Mathematical Model and FEM Solver}

In our method, each organ is modeled as a three-dimensional elastic object. Here we briefly introduce the formulation of linear elasticity and the finite element method. Assuming linear elasticity, the potential energy of an elastic body can be expressed as 


$$
E=\frac{1}{2} \int_{\Omega} \sigma^{T} \varepsilon d \Omega+\int_{\Omega} \mathbf{F u} d \Omega
$$

where $\mathbf{F}$ is the external force, $\mathbf{u}=[u, v, w]^{T}$ is the deformation vector, $\varepsilon$ is the strain, and $\sigma$ is the stress [11. When expressed in vector form, the strain is written as $\epsilon=\left[\frac{\partial u}{\partial x}, \frac{\partial v}{\partial y}, \frac{\partial w}{\partial z}, \frac{\partial u}{\partial y}+\frac{\partial v}{\partial x}, \frac{\partial v}{\partial z}+\frac{\partial w}{\partial y}, \frac{\partial u}{\partial z}+\frac{\partial w}{\partial x}\right]^{T}=\mathbf{L u}$, and the stress is a linear transformation of the strain, $\sigma=\left[\sigma_{x}, \sigma_{y}, \sigma_{z}, \tau_{x y}, \tau_{y z}, \tau_{x z}\right]^{T}=\mathbf{D} \varepsilon$, where $\mathbf{D}$ is a matrix defined by the material properties of the body. Assuming isotropic material properties, D is composed of two parameters: Young's modulus E, which measures the stiffness of a material, and Poisson's ratio $\nu$, which measures the tendency of contract in other directions when stretched in one direction.

Before we can find an optimal deformation $\mathbf{u}$ given the boundary conditions, we need a means to approximate derivatives. The finite element method is suitable for solving partial differential equations with non-regular domains. The domain is subdivided into polyhedral elements with vertices called nodes. The deformation field $\mathbf{u}$ in each element is approximated by a weighted sum $\hat{\mathbf{u}}=\sum_{i=1}^{n_{n}} N_{i}^{e l} \mathbf{u}_{i}^{e l}$, where $N_{i}^{e l}(\mathbf{x})$ is the shape function, $\mathbf{u}_{i}$ is the deformation vector at node $i$, and $n_{n}$ is the number of nodes. The superscript $e l$ indicates that the node numbering is local to each element. To represent the shape of organs, we use tetrahedral elements with linear shape functions, with $n_{n}=4$. The linear shape function $N_{i}$ is chosen such that it has value 1 at node $i$ and has value zero at all other nodes. Now we substitute $\hat{\mathbf{u}}$ for $\mathbf{u}$, take the derivative with respective to $\mathbf{u}_{i}^{e l}$, and drive it to zero:

$$
\int_{\Omega} \sum_{j=1}^{n_{n}} \mathbf{B}_{i}^{e l^{T}} \mathbf{D B}_{j} \mathbf{u}_{j}^{e l} d \Omega=-\int_{\Omega} \mathbf{F} N_{i}^{e l} d \Omega
$$

When expressed in matrix form, (2) becomes $\mathbf{K}^{e l} \mathbf{u}^{e l}=-\mathbf{F}^{e l}$, where matrix $\mathbf{K}^{e l}$ and vector $\mathbf{F}^{e l}$ are defined by $K_{i j}^{e l}=\int_{\Omega} \mathbf{B}_{i}^{e l^{T}} \mathbf{D} \mathbf{B}_{j}^{e l} d \Omega$ and $F_{i}^{e l}=\int_{\Omega} \mathbf{F} N_{i}^{e l} d \Omega$, respectively. The linear system is solved with Krylov subspace methods [17.

\subsection{Boundary Conditions for FEM-Based Simulation}

To solve (2), we need to specify boundary conditions. Two classes of boundary conditions are used in this work: one is the given external force $F_{i}$, and the other are the Dirichlet (first kind) boundary conditions [11, which specify the values of the deformation vectors at boundaries. External forces can be used to generate synthetic deformations, and Dirichlet boundary conditions can be given by surface matching algorithms for deformable registration. However, surface matching is not necessarily physically accurate.

To validate our model, we use a boundary condition based on organ-organ interactions: the deformation of the target organ is driven by contact forces between surrounding organs rather than the result of surface matching. We only match the surfaces of surrounding organs, such as the rectum and the bladder, instead of the surface of the target organ, such as the prostate, assuming the 
bones are fixed. Since the entire region of interest is simulated, the contact force between organs and the surrounding tissues will then make the target organ deform in a physically realistic way.

\subsection{Validation of Deformation Fields}

Validating a deformation field by a difference image of the deformed image and target image does not show 3D correspondence due to organ deformations. Therefore, we propose using the deformation field generated by a simulation to evaluate a registration algorithm. We begin with one image $I_{m}(\mathbf{x})$ and perform an FEM-based simulation given a set of boundary conditions. Let the resulting deformed image be $I_{m}^{\prime}(\mathbf{x})$ and the deformation field be a function $\mathbf{d}(\mathbf{x})$ that maps each point to a new location. Then we use another registration algorithm to register $I_{m}^{\prime}$ toward $I_{m}$ and generate a deformation field $\hat{\mathbf{d}}^{\prime}(\mathbf{x})$. While there can be infinitely many deformation fields that can register the two images, we are interested in finding the physically-accurate one, which should be the function inverse of $\mathbf{d}(\mathbf{x})$, to the extent that our FEM model is correct. Arbitrarily deformed images have been widely used for testing, but our method uses a physically-plausible deformation to achieve better physical accuracy.

\section{$3 \quad$ Experiments and Results}

Input surface meshes are built from hand-segmented CT images. After mesh simplification and smoothing [18, the entire region of interest is tetrahedralized with a three-dimensional Delaunay triangulator [19]. The organs include the bladder, rectum, and prostate, along with the bones. The surface and volumetric meshes are shown in Fig. 1]
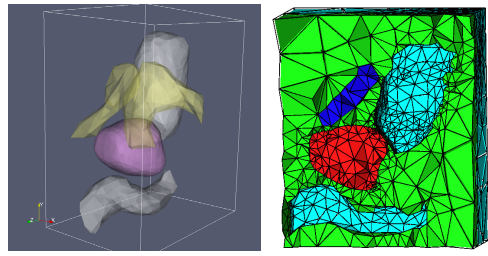

Fig. 1. The surface mesh (left) and a slice of tetrahedralized mesh (right)

The bones are fixed since no joints are included in the simulated region. The bladder and rectum are modeled as hollow objects. The prostate and surrounding tissues are modeled with isotropic elastic materials, which are defined by two parameters: Young's modulus $E$ and Poisson's ratio $\nu$. We set $E=40 \mathrm{kPa}$ and $\nu=0.4$ for the prostate, and $E=1 \mathrm{kPa}$ and $\nu=0.35$ for the pelvic tissue. These parameters can differ from patient to patient. The parameters for the prostate are adapted from Zhang et al. [20], and the values for surrounding tissues are optimized during the verification process. The assembly and solving of the linear system is based on the library PETSc [21, and the medical imaging library used is the Insight Toolkit (ITK) [22]. 


\subsection{Verification of Simulation}

In order to verify that our model is physically accurate, we conduct an experimental registration of the prostate with real CT images. In the experiment we would like to find a deformation field $\mathbf{d}$ to warp the moving image $I_{m}$ toward the target image $I_{t}$. Both images are segmented, and surface meshes of organs are extracted. Here we are interested in the correspondence of the prostate.

We apply a Dirichlet boundary condition on the rectum and the bladder to match their surfaces and perform the simulation. The resulting image and deformation field is shown in Fig. 2, where the red contour in the figure represents the prostate surface in $I_{t}$, and the blue one shows the surface before warping. The prostate matches reasonably well. The average distance to the target organ decreased from $0.44 \mathrm{~cm}$ to $0.17 \mathrm{~cm}$, and the standard deviation of the distance decreased from $0.24 \mathrm{~cm}$ to $0.08 \mathrm{~cm}$. The result supports the use of organ-organ interactions and indicates that our system produces physically plausible deformation suitable for validation of image registration.
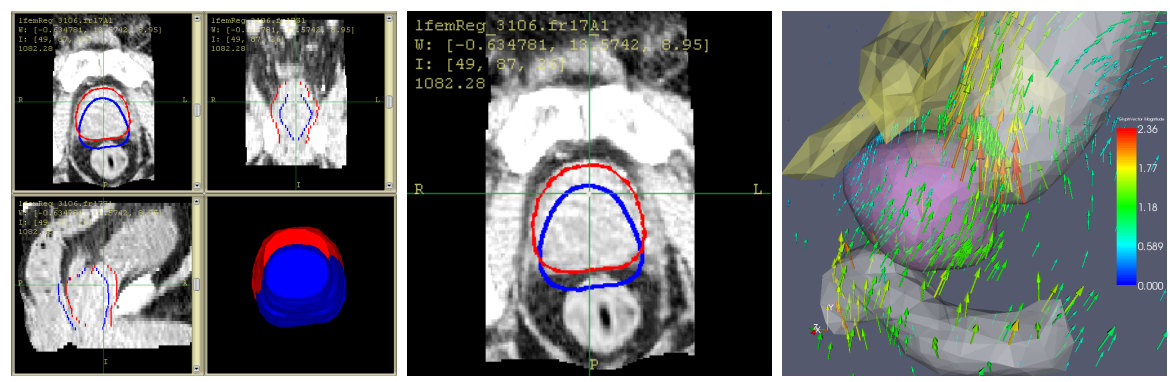

Fig. 2. Result of verification: left and center: image deformed by our simulator; red contour: prostate in $I_{t}$; blue contour: prostate in $I_{m}$; right: the resulting deformation

\subsection{Validation of Deformation Fields}

In the validation process, we register the synthesized image $I_{m}^{\prime}$ toward the original image $I_{m}$. Two optical-flow-based methods, the Demons method [1] and a level-set method [2] implemented in ITK [22], and a physically-based method, the viscous fluid flow method [10], are evaluated. We chose to test these methods because local features are harder to track around the prostate, and testing an FEM-based method against itself is not appropriate.

The $I_{m}^{\prime}$ for the first experiments is the result from Sect. 3.1 and the $I_{m}^{\prime}$ for the second experiment is generated by applying a constant inward pressure on the boundary of the bladder. The registered images and the deformation fields $\hat{\mathbf{d}}^{\prime}$ of the three methods composed with $\mathbf{d}$ are shown in Fig. 3 .

Ideally, the deformation fields $\mathbf{d}$ and $\hat{\mathbf{d}}^{\prime}$ should be the inverse mapping of each other, and therefore the composition should have values close to zero. We use the values $\epsilon_{a b s}=\operatorname{length}\left(\hat{\mathbf{d}}^{\prime}(\mathbf{d}(\mathbf{x}))\right)$ and $\epsilon_{r e l}=\operatorname{length}\left(\hat{\mathbf{d}}^{\prime}(\mathbf{d}(\mathbf{x}))\right) / \operatorname{length}(\mathbf{d}(\mathbf{x}))$ as the measures of absolute and relative error of $\hat{\mathbf{d}}^{\prime}$. Table 1 shows the errors in 


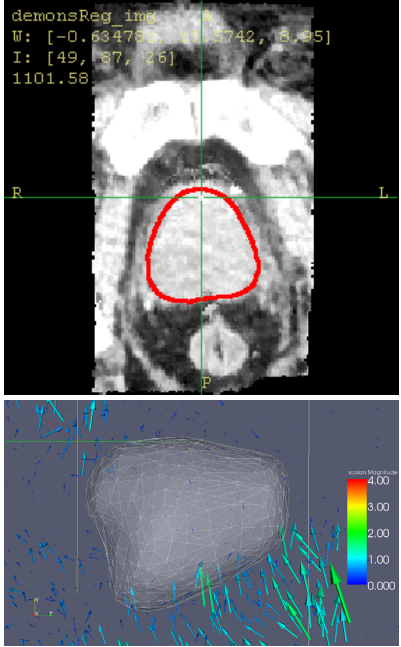

(a)

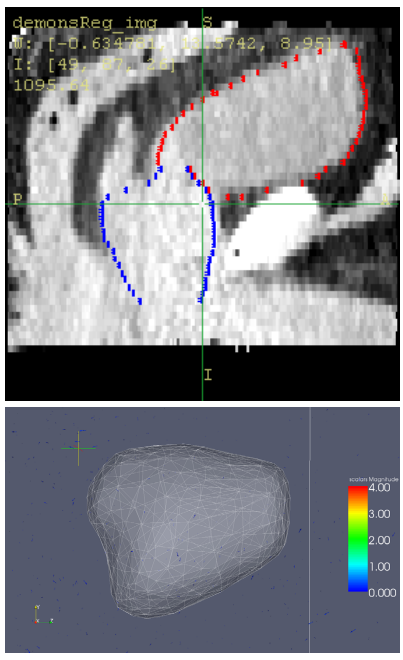

(d)

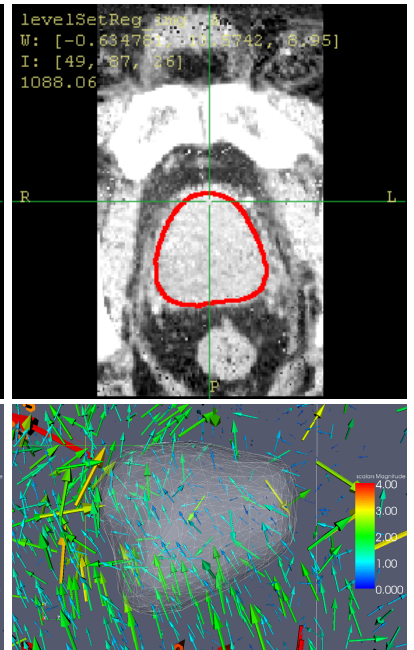

(b)

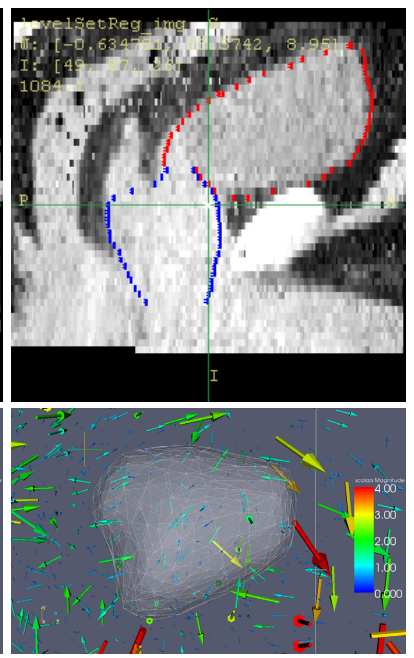

(e)

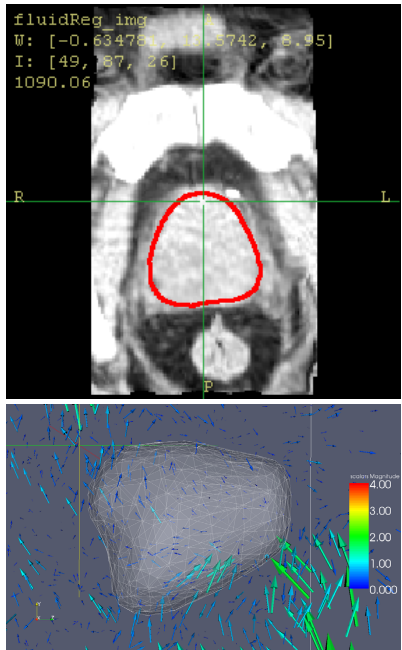

(c)

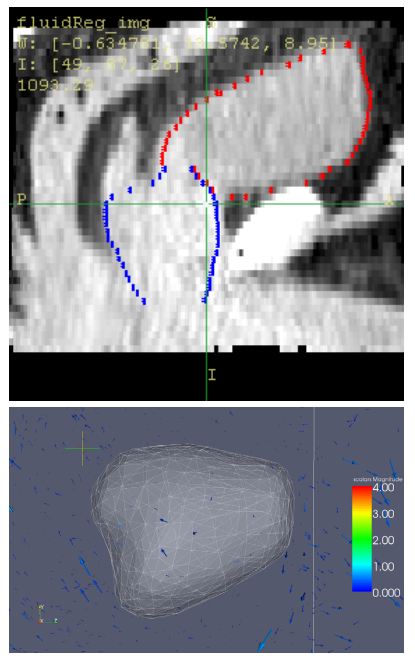

(f)

Fig. 3. Validation of the three registration methods. (a-c) the results of the Demons, level-set, and fluid flow method in the first experiment; (d-f) the results of the three method in the second experiment. The first and third row: registered images; the second and fourth row: composed deformation field.

the entire domain and inside the prostate. While all three methods generate well registered images, the errors in the deformation field vary. The Demons method produces the best results. The fluid flow method, although it is physically-based, does not perform better. This may due to the fact that the driving force is still derived from the image, and that human organs do not actually flow like fluid. The level-set method produces sharper images but has errors larger than 
Table 1. The mean (std. deviation) of $\epsilon_{a b s}$ and $\epsilon_{r e l}$ generated by the three methods

\begin{tabular}{|c|c|c|c||c|c|c|}
\hline & \multicolumn{3}{|c||}{ Experiment 1 } & \multicolumn{3}{c|}{ Experiment 2 } \\
\cline { 2 - 7 } & Demons & Level-Set & Fluid Flow & Demons & Level-Set & Fluid Flow \\
\hline \hline$\epsilon_{a b s}(\mathrm{~cm})$ & $0.26(0.41)$ & $1.10(0.91)$ & $0.37(0.37)$ & $0.05(0.03)$ & $0.72(1.00)$ & $0.17(0.16)$ \\
\hline$\epsilon_{\text {rel }}$ & $0.41(1.55)$ & $2.50(15.58)$ & $0.82(3.93)$ & $0.75(5.21)$ & $11.77(127.16)$ & $2.10(10.33)$ \\
\hline$\epsilon_{a b s}(\mathrm{~cm})$ in prostate & $0.16(0.18)$ & $1.12(0.57)$ & $0.30(0.19)$ & $0.04(0.03)$ & $0.35(0.36)$ & $0.04(0.03)$ \\
\hline$\epsilon_{\text {rel }}$ in prostate & $0.16(0.13)$ & $1.28(0.61)$ & $0.32(0.12)$ & $0.57(0.57)$ & $4.62(5.97)$ & $0.50(0.41)$ \\
\hline
\end{tabular}

the other two methods have. This may be a result of scaling the deformation vectors by a function of the difference of intensities in the two images. While the directions of the deformation may be correct, the magnitude may not be physically accurate. Zhong et al. 9] reported that the Demons method performs worse than a feature tracking method. The Demons method may not be as stable as the feature tracking or the fluid flow method when the deformation is very large, which is not the case in our experiments.

\section{Discussion}

We have presented a new method to validate a deformable registration method. While FEM-based methods are dependent on boundary conditions, we propose using inter-organ contact forces on target organs instead of specifying boundary conditions for them explicitly. A similar multi-organ method has been proposed [16], but we model the surrounding organs more accurately, and our deformations are not as extreme and is thus suitable for linear elasticity model. Our system is verified by a test registration, and we are able to register the target organ quite well without applying boundary conditions on it.

Our system provides physically accurate deformed images with known deformation fields for testing other image registration algorithms. Comparing a deformation against it provides results with a clear geometric interpretation. By comparison, the FEM approach of 9 is harder to interpret and lacks a definitive ground truth, but it can be used with pairs of real world images.

In the future, we would like to improve the mechanical model, such as by adding anisotropic thin layers and muscles, and apply it on different organs such as the the liver. Better collision detection algorithms may be incorporated into our system to handle self-collision of deformable models. We would also like to explore ways to evaluate the material properties of organs with simulations.

\section{Acknowledgment}

This work was supported in part by NCRR/NCI Grant R01 RR018615. We would like to thank the Army Research Office, the National Science Foundation, and Intel for their support. We would like to thank Gregg Tracton for providing the $\mathrm{CT}$ images and the surface models of the organs for the experiments. 


\section{References}

1. Thirion, J.P.: Image matching as a diffusion process: an analogy with maxwell's demons. Medical Image Analysis 2(3), 243-260 (1998)

2. Vemuri, B.C., Ye, J., Chen, Y., Leonard, C.M.: Image registration via level-set motion: applications to atlas-based segmentation. Medical image analysis 7, 1-20 (2003)

3. Shen, D., Davatzikos, C.: Hammer: Hierarchical attribute matching mechanism for elastic registration. IEEE Transactions on Medical Imaging 21(11), 1421-1439 (2002)

4. Lu, W., Chen, M.L., Olivera, G.H., Ruchala, K.J., Mackie, T.R.: Fast free-form deformable registration via calculus of variations. Physics in Medicine and Biology 49, 3067-3087 (2004)

5. Foskey, M., Davis, B., Goyal, L., Chang, S., Chaney, E., Strehl, N., Tomei, S., Rosenman, J., Joshi, S.: Large deformation three-dimensional image registration in image-guided radiation therapy. Physics in Medicine and Biology 50, 5869-5892 (2005)

6. Goshtasby, A.: Registration of images with geometric distortions. IEEE Transactions on Geoscience and Remote Sensing 26, 60-64 (1988)

7. Davis, M., Khotanzad, A., Flamig, D., Harms, S.: A physics-based coordinate transformation for 3-d image matching. IEEE Transactions on Medical Imaging 16, 317-328 (1997)

8. Kaus, M.R., Brock, K.K., Pekar, V., Dawson, L.A., Nichol, A.M., Jaffray, D.A.: Assessment of a model-based deformable image registration approach for radiation therapy planning. Int. J. Radiation Oncology Bio. Phys. 68(2), 572-580 (2007)

9. Zhong, H., Peters, T., Siebers, J.V.: FEM-based evaluation of deformable image registration for radiation therapy. Physics in Medicine and Biology 52, 4721-4738 (2007)

10. Christensen, G., Rabbitt, R.D., Miller, M.I.: Deformable templates using large deformation kinematics. IEEE Trans. Image Process. 5(10) (1996)

11. Zienkiewicz, O.C., Taylor, R.L.: The Finite Element Method, 6th edn., Butterworth-Heinemann (2005)

12. Park, K., Metaxas, D., Axel, L.: A finite element model for functional analysis of 4d cardiac-tagged mr images. In: Ellis, R.E., Peters, T.M. (eds.) MICCAI 2003. LNCS, vol. 2878, pp. 491-498. Springer, Heidelberg (2003)

13. Ferrant, M., Warfield, S.K., Guttmann, C.R.G., Mulkern, R.V., Jolesz, F.A., Kikinis, R.: 3d image matching using a finite element based elastic deformation model. In: Taylor, C., Colchester, A. (eds.) MICCAI 1999. LNCS, vol. 1679, pp. 202-209. Springer, Heidelberg (1999)

14. Ferrant, M., Warfield, S.K., Nabavi, A., Jolesz, F.A., Kikinis, R.: Registration of 3d intraoperative MR images of the brain using a finite element biomechanical model. In: Delp, S.L., DiGoia, A.M., Jaramaz, B. (eds.) MICCAI 2000. LNCS, vol. 1935, pp. 19-28. Springer, Heidelberg (2000)

15. Liang, J., Yan, D.: Reducing uncertainties in volumetric image based deformable organ registration. Medical Physics 30, 2116-2122 (2003)

16. Hensel, J.M., Menard, C., Chung, P.W.M., Milosevic, M.F., Kirilova, A., Moseley, J.L., Haider, M.A., Brock, K.K.: Development of multiorgan finite elementbased prostate deformation model enabling registration of endorectal coil magnetic resonance imaging for radiotherapy planning. Int. J. Radiation Oncology Bio. Phys. 68(5), 1522-1528 (2007) 
17. Kelley, C.T.: Iterative Methods for Linear and Nonlinear Equations, 1st edn. Society for Industrial Mathematics (1987)

18. Cignoni, P.: Meshlab., http://meshlab.sourceforge.net

19. Si, H.: Tetgen: A quality tetrahedral mesh generator and three-dimensional delaunay triangulator., http://tetgen.berlios.de/

20. Zhang, M., Nigwekar, P., Castaneda, B., Hoyt, K., Joseph, J.V., di Sant'Agnese, A., Messing, E.M., Strang, J.G., Rubens, D.J., Parker, K.J.: Quantitative characterization of viscoelastic properties of human prostate correlated with histology. In: Ultrasound in Medicine \& Biology (Corrected Proof)

21. Mathematics and Computer Science Division, Argonne National Laboratory: Portable, extensible toolkit for scientific computation., http://acts.nersc.gov/petsc/

22. Insight Software Consortium: Insight segmentation and registration toolkit (itk)., http://www.itk.org/ 